PROCEEDINGS OF THE

AMERICAN MATHEMATICAL SOCIETY

Volume 132, Number 4, Pages 945-953

S 0002-9939(03)07163-6

Article electronically published on September 22, 2003

\title{
SYMMETRIC WORD EQUATIONS IN TWO POSITIVE DEFINITE LETTERS
}

\author{
CHRISTOPHER J. HILLAR AND CHARLES R. JOHNSON
}

(Communicated by David R. Larson)

\begin{abstract}
For every symmetric ("palindromic") word $S(A, B)$ in two positive definite letters and for each fixed $n$-by- $n$ positive definite $B$ and $P$, it is shown that the symmetric word equation $S(A, B)=P$ has an $n$-by- $n$ positive definite solution $A$. Moreover, if $B$ and $P$ are real, there is a real solution $A$. The notion of symmetric word is generalized to allow non-integer exponents, with certain limitations. In some cases, the solution $A$ is unique, but, in general, uniqueness is an open question. Applications and methods for finding solutions are also discussed.
\end{abstract}

\section{INTRODUCTION}

A generalized word ( $g$-word, for short) $W=W(A, B)$ in two letters $A$ and $B$ is an expression of the form

$$
W=A^{p_{1}} B^{q_{1}} A^{p_{2}} B^{q_{2}} \cdots A^{p_{k}} B^{q_{k}} A^{p_{k+1}}
$$

in which the exponents $p_{i}$ and $q_{i}$ are real numbers such that $p_{i}, q_{i} \neq 0, i=1, \ldots, k$, and $p_{k+1}$ is an arbitrary real number. We call $k$ the class number of $W$. The reversal of the g-word $W$ is $W^{*}=A^{p_{k+1}} B^{q_{k}} A^{p_{k}} \cdots B^{q_{2}} A^{p_{2}} B^{q_{1}} A^{p_{1}}$ and a g-word is symmetric if it is identical to its reversal (in other contexts, the name "palindromic" is also used). We will call a g-word, $W, A$-positive (A-negative) if all exponents of $A$ in $W$ are positive (negative).

We are interested in the matrices that result when the two letters are (independent) positive definite (complex Hermitian) $n$-by- $n$ matrices (PD, for short). For convenience, the letters $A, B$ will also represent the substituted PD matrices (the context will make the distinction clear). To make sure that $W$ is well-defined after substitution, we take primary PD powers (see [3 p. 433] and [3, p. 413]). That is, given $p \in \mathbb{R} \backslash\{0\}$, a unitary matrix $U$, and a nonnegative diagonal matrix $D$, we have $\left(U D U^{*}\right)^{p}=U D^{p} U^{*}$.

Received by the editors June 21, 2002 and, in revised form, November 20, 2002.

2000 Mathematics Subject Classification. Primary 15A24, 15A57; Secondary 15A18, 15A90.

Key words and phrases. Positive definite matrix, generalized word, symmetric word equation.

This research was conducted, in part, during the summer of 1999 at the College of William and Mary's Research Experiences for Undergraduates program and was supported by NSF REU grant DMS-96-19577.

The work of the first author is supported under a National Science Foundation Graduate Research Fellowship. 
Our primary interest will be matrix equations involving $A$-positive symmetric g-words.

Definition 1.1. A symmetric word equation is an equation, $S(A, B)=P$, in which $S(A, B)$ is an $A$-positive symmetric g-word. If $B$ and $P$ are given positive definite matrices, any positive definite matrix $A$ for which the equation holds is called a solution to the symmetric word equation.

A symmetric word equation will be called solvable if there exists a solution for every pair of positive definite $n$-by-n $B, P$. Moreover, if each such pair gives rise to a unique solution, the equation will be called uniquely solvable. It is clear that the (unique) solvability of $A$-positive word equations implies the same as for $A$-negative equations (by replacing $A$ with $A^{-1}$ ), and hence, no generality is lost in Definition 1.1. As we shall soon see, the restriction of the definition above to $A$-positive words is important.

We first encountered symmetric word equations while studying a trace conjecture 4] in the case of words with positive integral powers. The conjecture is

Conjecture 1.2. A word has positive trace for every pair of real positive definite matrices if and only if the word is symmetric or a product (juxtaposition) of 2 symmetric words.

It turns out that for each solvable symmetric word equation, we can identify an infinite class of words that admit real PD matrices $A$ and $B$ giving those words a negative trace. Our investigation of these equations, although useful for our methods in 4], shows that they have a rich structure of their own, some of which we explore here. (See Section 7 for a generalization of the notion of "symmetric word equation" defined above.) A natural question to ask is if it is necessary to focus attention on symmetric g-words. We remark that it can be shown that symmetric g-words are the only g-words that are positive definite for all positive definite matrices $A, B$. In light of Definition 1.1, our restriction seems appropriate.

\section{SYMmetric WORDS}

Recall that two $n$-by- $n$ matrices $X$ and $Y$ are said to be congruent if there is an invertible $n$-by- $n$ matrix $Z$ such that $Y=Z^{*} X Z$; and that congruence on Hermitian matrices preserves inertia (the ordered triple consisting of the number of positive, negative, and zero eigenvalues) and, thus, positive definiteness [2, p. 223]. A symmetric word of class $k$ in two positive definite matrices is congruent to one of class $k-1$, iteration of which implies congruence to the "center," class 0 , positive definite matrix. We conclude that

Lemma 2.1. A symmetric g-word in two positive definite matrices is positive definite.

It is natural to ask if the map from the set of $\mathrm{PD}$ matrices to itself given by $h: A \mapsto S(A, B)$ is surjective. Our main result answers this in the affirmative.

Theorem 2.2. Every symmetric word equation is solvable.

We remark that the statement is not generally valid if the definition of "symmetric word equation" is widened to allow mixed sign powers of $A$, even in the case $n>1$. Let $I$ denote the $n$-by- $n$ identity matrix and let $B \neq I$ be an $n$-by- $n$ PD matrix. Then, the equation $A^{-1} B A^{2} B A^{-1}=I$ has no PD solution. For if there 
were a PD solution $A$, then $A B A^{-1}=U$, for some unitary $U$. Hence, $B=A^{-1} U A$ has eigenvalues on the unit disc. The only positive definite matrix for which this is so is $B=I$.

We believe that $h$ is also injective, but this is proven only in special cases. This is

Conjecture 2.3. Every symmetric word equation is uniquely solvable.

\section{Some SPECific Equations}

In what follows, we shall say that two symmetric word equations are (uniquely) equivalent if for each, its (unique) solvability implies the (unique) solvability of the other. For instance, the equations $A B A^{2} B A=P$ and $A B A=P$ are uniquely equivalent because $\mathrm{PD}$ matrices have unique $\mathrm{PD}$ square roots. As a more subtle example, the equations $A^{2} B A B A^{2}=P$ and $A B A^{3} B A=P$ are seen to be uniquely equivalent by setting $A=B^{-1 / 2} X B^{-1 / 2}$ in $A^{2} B A B A^{2}=P$. Additionally, the same substitution gives us that for any integer $k>2, A^{2}(B A)^{k-2} B A^{2}=P$ and $A B A^{k} B A=P$ are uniquely equivalent.

It is an easy exercise that the facts below follow from our definition of unique equivalence:

(1) For $r>0$ and $s \neq 0$, the equations $S\left(A^{r}, B^{s}\right)=P$ and $S(A, B)=P$ are uniquely equivalent.

(2) For each positive integer $k, S(A, B)^{k}=P$ and $S(A, B)=P$ are uniquely equivalent.

(3) For $s$ real, the equations $B^{s} S(A, B) B^{s}=P$ and $S(A, B)=P$ are uniquely equivalent.

The simplest examples of symmetric word equations are $\left\{A^{q}=P, q \in \mathbb{R} \backslash\{0\}\right\}$. These satisfy Conjecture 2.3 by uniqueness of $\mathrm{PD} q^{\text {th }}$ roots. More interesting is the first nontrivial equation, $A B A=P$. This equation has arisen in other contexts [1]. We indicate some aspects of this equation that will be useful to us.

Theorem 3.1. The equation $A B A=P$ has a unique solution for each pair of $P D$ matrices $B, P$. Moreover, the unique $P D$ matrix $A$ is given by

$$
A=B^{-1 / 2}\left(B^{1 / 2} P B^{1 / 2}\right)^{1 / 2} B^{-1 / 2} .
$$

Proof. Assume that $P$ and $B$ are given positive definite matrices and that $A$ is a PD solution to $A B A=P$. Set $X=B^{1 / 2} A B^{1 / 2}$, which is PD by Lemma 2.1, so that $A=B^{-1 / 2} X B^{-1 / 2}$. Then,

$$
P=B^{-1 / 2} X B^{-1 / 2} B B^{-1 / 2} X B^{-1 / 2}=B^{-1 / 2} X^{2} B^{-1 / 2} .
$$

Therefore, $X^{2}=B^{1 / 2} P B^{1 / 2}$, from which it follows that $X$ is uniquely determined as $\left(B^{1 / 2} P B^{1 / 2}\right)^{1 / 2}$. Hence, $A$ must be $B^{-1 / 2}\left(B^{1 / 2} P B^{1 / 2}\right)^{1 / 2} B^{-1 / 2}$. Finally, substituting this positive definite $A$ (by Lemma 2.1 again) into the original equation does verify that it is a solution.

As in [1] given two $\mathrm{PD}$ matrices $C$ and $D$ we will denote $C \# D$ as the PD matrix,

$$
C \# D=C^{1 / 2}\left(C^{-1 / 2} D C^{-1 / 2}\right)^{1 / 2} C^{1 / 2},
$$

the so-called geometric mean of $C$ and $D$. Notice that from Theorem 3.1, $A B A=P$ has the unique solution $A=\left(B^{-1}\right) \# P$. Writing this equation (by inverting) as $B^{-1}=A P^{-1} A$, we also have that $A=P \#\left(B^{-1}\right)$, and from this comes the not so obvious fact that $C \# D=D \# C$. 


\section{Fixed POINTS AND A SEQUENCE LEMma}

For more complex symmetric word equations, it is not clear that there should be an explicit formula for a solution, as in Theorem 3.1. We do not know one, for example, for $A^{2} B A B A^{2}=P$. Since our proof of the solvability of this equation (and all others) will use fixed-point theory, we record a useful theorem of Brouwer [5].

Theorem 4.1 (Brouwer's fixed point theorem). If $M$ is a compact, convex subset of a finite-dimensional Banach space and if $f: M \rightarrow M$ is a continuous function, then there is a fixed point, $p$, for $f$ in $M$.

We will be using the spectral matrix norm throughout (see [2 p. 295]). This norm is useful because for positive semidefinite $A$, it is just the largest eigenvalue of A. Brouwer's result is an important ingredient in the proof of Theorem 2.2. Before proving Theorem 2.2, we record the following.

Lemma 4.2. Suppose $\left\{A_{k}\right\}$ is a convergent sequence of positive definite matrices. Then there is a subsequence $\left\{A_{k_{j}}\right\}_{j=1}^{\infty}$ such that

$$
\lim _{j \rightarrow \infty} \frac{\left(A_{k_{j}}+I / k_{j}\right)^{-1}}{\left\|\left(A_{k_{j}}+I / k_{j}\right)^{-1}\right\|}
$$

exists.

Proof. Set $A_{k}=U_{k} D_{k} U_{k}^{*}$ for unitary $U_{k}$ and $D_{k}=\operatorname{diag}\left(\lambda_{1 k}, \lambda_{2 k}, \ldots, \lambda_{n k}\right)$ in which $0<\lambda_{1 k} \leq \lambda_{2 k} \leq \ldots \leq \lambda_{n k}$ are the eigenvalues of $A_{k}$. For each $m \in\{1, \ldots, n\}$, define new sequences

$$
h_{m}(k)=\frac{\lambda_{1 k}+1 / k}{\lambda_{m k}+1 / k} .
$$

Notice that $h_{1}(k)=1$ for all $k$. We now show by induction that there exists a subsequence, $\left\{k_{j}\right\}_{j=1}^{\infty}$, such that each of $\left\{h_{m}\left(k_{j}\right)\right\}_{j=1}^{\infty}$ converges. To simplify matters later, we first assume that $\left\{U_{k}\right\}$ converges to a unitary $U$ by passing to a subsequence (the set of unitary matrices is compact).

Let $M \in\{1, \ldots, n\}$ be such that there exists a subsequence $\left\{k_{j}\right\}_{j=1}^{\infty}$ making each of $\left\{h_{1}\left(k_{j}\right)\right\}_{j=1}^{\infty}, \ldots,\left\{h_{M}\left(k_{j}\right)\right\}_{j=1}^{\infty}$ converge. Such an $M$ clearly exists (e.g. $M=1$ ). If $M=n$, then there is nothing to prove. Otherwise, examine the inequality

$$
0<h_{M+1}\left(k_{j}\right)=\frac{\lambda_{1 k_{j}}+1 / k_{j}}{\lambda_{(M+1) k_{j}}+1 / k_{j}} \leq 1
$$

which holds for all $j$. Since this is a bounded sequence, choose a subsequence of the sequence, $\left\{k_{j}\right\}_{j=1}^{\infty}$, making this ratio converge to some nonnegative number. This will not alter the convergence of the first $M$ sequences. This completes the induction.

We conclude that there exists a subsequence, $\left\{k_{j}\right\}_{j=1}^{\infty}$, such that

$$
\lim _{j \rightarrow \infty} \frac{\lambda_{1 k_{j}}+1 / k_{j}}{\lambda_{m k_{j}}+1 / k_{j}}=h_{m} \geq 0 .
$$


We now claim that $\lim _{j \rightarrow \infty} \frac{\left(A_{k_{j}}+I / k_{j}\right)^{-1}}{\left\|\left(A_{k_{j}}+I / k_{j}\right)^{-1}\right\|}$ exists. But the sequence in question is just

$$
U_{k_{j}}\left(\begin{array}{ccc}
\frac{\lambda_{1 k_{j}}+1 / k_{j}}{\lambda_{1 k_{j}}+1 / k_{j}} & \cdots & 0 \\
\vdots & \ddots & \vdots \\
0 & \cdots & \frac{\lambda_{1 k_{j}}+1 / k_{j}}{\lambda_{n k_{j}}+1 / k_{j}}
\end{array}\right) U_{k_{j}}^{*},
$$

which by construction has the limit

$$
U\left(\begin{array}{ccc}
1 & \cdots & 0 \\
\vdots & \ddots & \vdots \\
0 & \cdots & h_{n}
\end{array}\right) U^{*}
$$

This proves the lemma.

\section{All symmetric Word equations are solvable}

We may now prove the main result.

Proof of Theorem 2.2. Notice (using (1), (3) above) that it is equivalent to study symmetric word equations of the form $A S(A, B) A=P$, where $S(A, B)$ is a symmetric g-word beginning and ending with a power of $A$. For instance, the equation $A^{1 / 2} B A B A^{1 / 2}=P$ is (uniquely) equivalent to $A\left(A B A^{4} B A\right) A=P$. The convex, compact set to which we apply Brouwer's theorem is

$$
M=\{A \mid A \text { is positive semidefinite and }\|A\| \leq 1\} .
$$

Define $S_{A}$ to denote the sum of all the powers of $A$ in $S(A, B)$. Also, let $S_{-B}$ $\left(S_{B}\right)$ denote the sum of all negative (positive) powers of $B$ in $S(A, B)$. Let $k$ be a positive integer. Define a function $f_{k}$ on the set $M$ as follows:

$$
\begin{aligned}
f_{k}(X) & =\frac{P \#\left(S(X+I / k, B)^{-1}\right)}{g_{k}(X)} \\
& =\frac{P^{1 / 2}\left(P^{-1 / 2} S(X+I / k, B)^{-1} P^{-1 / 2}\right)^{1 / 2} P^{1 / 2}}{g_{k}(X)}
\end{aligned}
$$

where $g_{k}: M \rightarrow \mathbb{R}^{+}$is defined by

$$
g_{k}(X)=\|P\|\left\|P^{-1}\right\|^{1 / 2}\|B\|^{S_{-B} / 2}\left\|B^{-1}\right\|^{S_{B} / 2}\left\|(X+I / k)^{-1}\right\|^{S_{A} / 2} .
$$

From the properties of the spectral matrix norm and the fact that $S(C, D)^{-1}=$ $S\left(C^{-1}, D^{-1}\right)$, it follows that $f_{k}$ is bounded by 1 , that is,

$$
\left\|f_{k}(X)\right\| \leq \frac{\|P\|\left\|P^{-1}\right\|^{1 / 2}\|B\|^{S_{-B} / 2}\left\|B^{-1}\right\|^{S_{B} / 2}\left\|(X+I / k)^{-1}\right\|^{S_{A} / 2}}{g_{k}(X)}=1
$$

We should note that in the 1-by-1 case, we simply have $f_{k}(X)=1$, so that the unique fixed point for $f_{k}(X)$ is given by $X=1$. More generally, from the properties of the geometric mean and since $k>0$, it is clear that $f_{k}(X)$ is positive definite. Hence, $f_{k}(X) \in M$. From the discussion of primary matrix functions [3, p. 433] for normal matrices, it is seen that for $q \neq 0, Y^{q}$ is a continuous function from the 
set of positive definite matrices into itself. Therefore, $f_{k}$ is also continuous on $M$ (it is made up of compositions and products of continuous functions).

Now, apply Brouwer's fixed point theorem to give us $A_{k}=f_{k}\left(A_{k}\right)$. Because $f_{k}$ is always positive definite, we must have that $A_{k}$ is nonsingular. Hence, from the properties of the geometric mean (Theorem 3.1), we have

$$
g_{k}\left(A_{k}\right)^{2} A_{k} S\left(A_{k}+I / k, B\right) A_{k}=P .
$$

Because $\left\{A_{k}\right\}_{k=1}^{\infty}$ is an infinite, bounded sequence of PD matrices, there is a subsequence that converges to a positive semidefinite matrix $T$. We will therefore assume that $\left\{A_{k}\right\}$ converges. If $T$ is actually positive definite, then we have

$$
P=\lim _{k \rightarrow \infty} g_{k}\left(A_{k}\right)^{2} A_{k} S\left(A_{k}+I / k, B\right) A_{k}=T S(T, B) T g_{\infty}(T)^{2},
$$

in which $g_{\infty}(T)=\|P\|\left\|P^{-1}\right\|^{1 / 2}\|B\|^{S_{-B} / 2}\left\|B^{-1}\right\|^{S_{B} / 2}\left\|T^{-1}\right\|^{S_{A} / 2}$. Then, it is easily seen that

$$
A=T \cdot g_{\infty}(T)^{2 /\left(2+S_{A}\right)}
$$

is our desired PD solution.

Since (5.1) has no limit interpretation if $T$ is singular, we now show that $T$ is necessarily positive definite. For each $k$, form the decomposition, $A_{k}=U_{k} D_{k} U_{k}^{*}$, with unitary $U_{k}$ and $D_{k}=\operatorname{diag}\left(\lambda_{1 k}, \lambda_{2 k}, \ldots, \lambda_{n k}\right)$, in which $0<\lambda_{1 k} \leq \lambda_{2 k} \leq \ldots \leq$ $\lambda_{n k}$ are the eigenvalues of $A_{k}$. Suppose that $\left\{A_{k}\right\}$ converges to a singular matrix $T$ with $0 \leq \lambda_{1} \leq \lambda_{2} \leq \ldots \leq \lambda_{n}$ being the eigenvalues of $T$. Since $T$ is assumed to be singular, let $m \geq 1$ be such that $\left\{\lambda_{1}, \lambda_{2}, \ldots, \lambda_{m}\right\}$ are the zero eigenvalues of $T$. Set $c=\|P\|^{2}\left\|P^{-1}\right\|\|B\|^{S_{-B}}\left\|B^{-1}\right\|^{S_{B}}$, and examine the equality (following from inverting equation (5.1))

$$
\left\|\left(A_{k}+I / k\right)^{-1}\right\|^{-S_{A}} S\left(\left(A_{k}+I / k\right)^{-1}, B^{-1}\right)=c A_{k} P^{-1} A_{k},
$$

which is just

$$
S\left(\frac{\left(A_{k}+I / k\right)^{-1}}{\left\|\left(A_{k}+I / k\right)^{-1}\right\|}, B^{-1}\right)=c A_{k} P^{-1} A_{k} .
$$

From Lemma 4.2, there is a subsequence $\left\{A_{k_{j}}\right\}_{j=1}^{\infty}$ such that $\left\{U_{k_{j}}\right\}_{j=1}^{\infty}$ converges to some unitary $U$ and such that $\lim _{j \rightarrow \infty} \frac{\left(A_{k_{j}}+I / k_{j}\right)^{-r}}{\left\|\left(A_{k_{j}}+I / k_{j}\right)^{-1}\right\|^{r}}$ exists for all $r>0$. Moreover, this limit is equal to

$$
U\left(\begin{array}{ccc}
1 & \cdots & 0 \\
\vdots & \ddots & \vdots \\
0 & \cdots & h_{n}^{r}
\end{array}\right) U^{*}
$$

for some $h_{2}, h_{3}, \ldots, h_{n} \geq 0$. If $h_{2}, h_{3}, \ldots, h_{n}$ are all nonzero, then the limit as $j \rightarrow \infty$ of

$$
S\left(\frac{\left(A_{k_{j}}+I / k_{j}\right)^{-1}}{\left\|\left(A_{k_{j}}+I / k_{j}\right)^{-1}\right\|}, B^{-1}\right)
$$

is invertible, while the limit of $c A_{k_{j}} P^{-1} A_{k_{j}}$ is singular. Whence, $h_{t}=0$ for some $t \in\{2,3, \ldots, n\}$. Moreover, if $s>t$, then $h_{s}=0$ as well, this coming from the fact 
that

$$
h_{t}\left(k_{j}\right)=\frac{\lambda_{1 k_{j}}+1 / k_{j}}{\lambda_{t k_{j}}+1 / k_{j}} \geq \frac{\lambda_{1 k_{j}}+1 / k_{j}}{\lambda_{s k_{j}}+1 / k_{j}}=h_{s}\left(k_{j}\right)
$$

by our ordering of the eigenvalues of $A_{k}$. So assume that $t$ is the largest element of $\{1,2, \ldots, n\}$ such that $h_{t} \neq 0$. Then, our limit (5.3) looks like

$$
L=U\left(\begin{array}{cc}
E_{h}^{r} & 0 \\
0 & 0
\end{array}\right) U^{*}=U E^{r} U^{*}
$$

in which $E_{h}=\operatorname{diag}\left(1, h_{2}, \ldots, h_{t}\right)$ is positive and $E=\operatorname{diag}\left(1, h_{2}, \ldots, h_{t}, 0, \ldots, 0\right)$. Now, set $\widetilde{B}=U^{*} B^{-1} U$. Then, the left-hand side of (5.2) (within the subsequence above) converges to

$$
S\left(U E U^{*}, B^{-1}\right)=U E^{p_{1}} \widetilde{B}^{q_{1}} E^{p_{2}} \widetilde{B}^{q_{2}} \cdots \widetilde{B}^{q_{2}} E^{p_{2}} \widetilde{B}^{q_{1}} E^{p_{1}} U^{*} .
$$

We claim (5.4) has the form

$$
U\left(\begin{array}{cc}
H & 0 \\
0 & 0
\end{array}\right) U^{*}
$$

for some PD $t$-by- $t$ matrix $H$. Indeed, the center matrix in (5.4) is of the form $E^{p}$ or $E^{p} \widetilde{B}^{q} E^{p}$, each being the direct sum of a $t$-by- $t$ PD matrix and an $(n-t)$-by- $(n-t)$ zero matrix. We now induct on the form of (5.4). Assume that $E^{p_{d}} \widetilde{B}^{q_{d}} \cdots \widetilde{B}^{q_{d}} E^{p_{d}}$ is a direct sum of a $t$-by- $t$ PD matrix $H_{d}$ with a zero matrix. Form the partition,

$$
\widetilde{B}^{q_{d-1}}=\left(\begin{array}{ll}
B_{11} & B_{12} \\
B_{21} & B_{22}
\end{array}\right)
$$

in which $B_{11}$ is a $t$-by- $t \mathrm{PD}$ matrix, $B_{22}$ is an $(n-t)$-by- $(n-t) \mathrm{PD}$ matrix and $B_{12}=B_{21}^{*}$ (see [2, p. 472]). Then,

$$
\begin{aligned}
E^{p_{d-1}} \widetilde{B}^{q_{d-1}}\left(\begin{array}{cc}
H_{d} & 0 \\
0 & 0
\end{array}\right) \widetilde{B}^{q_{d-1}} E^{p_{d-1}} & =E^{p_{d-1}}\left(\begin{array}{ll}
B_{11} H_{d} B_{11} & B_{11} H_{d} B_{12} \\
B_{21} H_{d} B_{11} & B_{21} H_{d} B_{12}
\end{array}\right) E^{p_{d-1}} \\
& =\left(\begin{array}{cc}
E_{h}^{p_{d-1}} B_{11} H_{d} B_{11} E_{h}^{p_{d-1}} & 0 \\
0 & 0
\end{array}\right)
\end{aligned}
$$

is of the desired form, completing the induction.

Examine now the right-hand side of $(5.2), c A_{k_{j}} P^{-1} A_{k_{j}}$, which converges to $c T P^{-1} T$. Since the left-hand side limit by above has rank $t>0, T$ cannot have all its eigenvalues equal to 0 . Whence, we can write this limit as $c U D U^{*} P^{-1} U D U^{*}$, in which $D=\left(\begin{array}{ll}0 & 0 \\ 0 & \Lambda\end{array}\right)$ and $\Lambda$ is the positive diagonal matrix, $\operatorname{diag}\left(\lambda_{m+1}, \ldots, \lambda_{n}\right)$. Setting these two expressions equal gives us

$$
c\left(\begin{array}{cc}
0 & 0 \\
0 & \Lambda
\end{array}\right) U^{*} P^{-1} U\left(\begin{array}{cc}
0 & 0 \\
0 & \Lambda
\end{array}\right)=\left(\begin{array}{cc}
H & 0 \\
0 & 0
\end{array}\right) .
$$

Of course, this equality is impossible. We simply compare the two $(n, n)$ entries of the left-hand and right-hand sides to arrive at a contradiction (one is positive, the other zero). Hence, $T$ is invertible, completing the proof.

We remark that if $P$ and $B$ are chosen to be real, then the proof above shows that the solution $A$ may be chosen to be real as well. 


\section{Approximate solutions}

We now make some remarks about finding approximate solutions to symmetric word equations. As a first approach one would hope that an iteration of the function defined in the proof of Theorem 2.2 would give rise to approximate solutions. Unfortunately, experimentation shows this not to be the case. We detail another method that has been quite effective in practice at finding solutions (in the case of positive integral powers) and in verifying our conjecture of unique solvability.

Given a symmetric word $S(A, B)$ with positive integral exponents, start with an initial PD matrix $A_{0}$ (usually $I$ ), and expand the expression $S\left(A_{0}+D, B\right)$. Consider the formal sum, $S^{\prime}\left(A_{0}+D, B\right)$, of the terms in this expansion with at most a single $D$. Now, solve the linear system

$$
S^{\prime}\left(A_{0}+D, B\right)=P
$$

for the matrix $D$ and set $A_{1} \leftarrow A_{0}+D$. Repeating this process gives our algorithm. As a simple example, the repeated equations for $A B A=P$ are given by

$$
\begin{gathered}
A_{i-1} B D+D B A_{i-1}=P-A_{i-1} B A_{i-1}, \\
A_{i}=A_{i-1}+D .
\end{gathered}
$$

Curiously enough, there seems to be no guarantee in general that these $A_{i}$ will be positive definite, nor is it clear that the linear system for $D$ above will always have a solution. Nonetheless, experimentation has shown that these iterations always converge to the same PD solution regardless of the initial starting point.

\section{A generalization}

We close by noting a generalization of Theorem 2.2 to a larger class of matrix equations. If $C_{1}, \ldots, C_{m}$ is any list of $m$ invertible $n$-by- $n$ matrices and $W\left(A ; C_{1}, \ldots, C_{m}\right)=A^{p_{1}} C_{1} A^{p_{2}} C_{2} \cdots C_{2}^{*} A^{p_{2}} C_{1}^{*} A^{p_{1}}$ is such that $W\left(A ; C_{1}, \ldots, C_{m}\right)^{*}$ $=W\left(A ; C_{1}, \ldots, C_{m}\right)$, we call $W$ a generalized symmetric word. It is again an elementary exercise in congruence that, if $A$ is $\mathrm{PD}$ and $W$ is a generalized symmetric word, then $W\left(A ; C_{1}, \ldots, C_{m}\right)$ is positive definite. For a given $\mathrm{PD}$ matrix $P$ and invertible $C_{1}, \ldots, C_{m}$ we call

$$
W\left(A ; C_{1}, \ldots, C_{m}\right)=P
$$

with each $p_{i}>0$, a generalized symmetric word equation in the $\mathrm{PD}$ variable $A$. Though we are not motivated by any particular application, generalized symmetric word equations are natural to consider on a theoretical level. Our proof of Theorem 2.2 goes over directly to generalized symmetric word equations, except that a further technical condition on the $C_{i}$ 's is needed. That condition is that the leading principal minors of any unitary similarity of $C_{i}^{-1}$ should be nonzero. Of course, this means that any principal minor of any unitary similarity of $C_{i}^{-1}$ should be nonzero for $i=1, \ldots, m$. Using elementary facts about the field of values of an $n$-by- $n$ matrix $C$ :

$$
F(C)=\left\{x^{*} C x \mid x^{*} x=1, x \in \mathbb{C}^{n}\right\},
$$

see chapter 1 of [3], the latter condition may easily be seen to be equivalent to the statement that $0 \notin F(C)$. For purposes of this work, we call such a matrix completely invertible. Of course, any power of a positive definite matrix $B$ is completely invertible, and this is what is essential in the proof of Theorem 2.2. For generalized 
symmetric word equations, the analysis of (5.4) remains valid because any partition as in (5.5) will have an invertible leading principle submatrix. We conclude

Theorem 7.1. For completely invertible $C_{1}, \ldots, C_{m}$, the generalized symmetric word equation

$$
W\left(A ; C_{1}, \ldots, C_{m}\right)=P
$$

is solvable for any positive definite $P$.

We have no example showing that complete invertibility cannot be replaced by invertibility.

\section{Acknowledgement}

The authors would like to thank Scott Armstrong for a careful reading of a preliminary version of this manuscript.

\section{REFERENCES}

1. T. Ando, On the Arithmetic-Geometric-Harmonic-Mean Inequalities for Positive Definite Matrices, Linear Algebra Appl., 52/53 (1983), 31-37. MR 84j:15016

2. R. Horn and C. R. Johnson, Matrix Analysis, Cambridge University Press, Cambridge, 1985. MR 87e:15001

3. R. Horn and C. R. Johnson, Topics in Matrix Analysis, Cambridge University Press, Cambridge, 1991. MR 92e:15003

4. C. R. Johnson and C. Hillar, Eigenvalues of Words in Two Positive Definite Letters, SIAM J. Matrix Anal. Appl., 23 (2002), 916-928. MR 2003e:81071

5. E. Zeidler, Applied Functional Analysis: Applications to Mathematical Physics, Applied Mathematical Sciences, Vol. 108, Springer-Verlag, New York, 1995. MR 96i:00005

Department of Mathematics, University of California, Berkeley, California 94720

E-mail address: chillar@math.berkeley.edu

Department of Mathematics, College of William and Mary, Williamsburg, Virginia 23187-8795

E-mail address: crjohnso@math.wm.edu 